\title{
Best PEEP trials are dependent on tidal volume
}

\author{
Andrew C. McKown ${ }^{*}$, Matthew W. Semler and Todd W. Rice
}

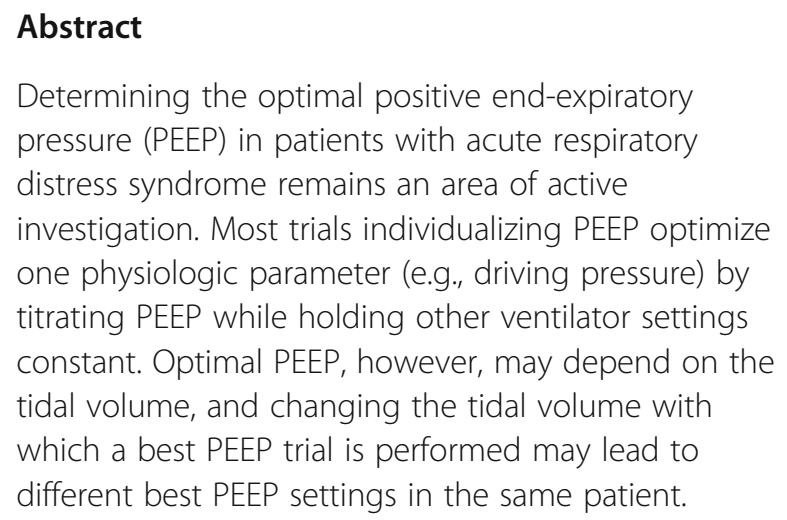

Trial registration: ClinicalTrials.gov, NCT02871102.

Registered on 12 August 2016.

Positive end-expiratory pressure (PEEP) may mitigate ventilator-induced lung injury in acute respiratory distress syndrome (ARDS) by recruiting collapsed alveolar units, thereby reducing stress raisers and minimizing atelectrauma [1]. Excessive PEEP, however, may cause barotrauma and biotrauma from alveolar hyperdistension. Many studies have attempted to identify the best PEEP for individual patients [2], including the recent Alveolar Recruitment for ARDS Trial (ART) which reported a higher mortality with use of a recruitment maneuver and titrated PEEP compared with use of lower PEEP [3].

We hypothesized that best PEEP, as selected by respiratory system compliance $\left(C_{R S}\right)$ or driving pressure (end-inspiratory plateau pressure minus PEEP), is contingent upon the tidal volume $\left(\mathrm{V}_{\mathrm{T}}\right)$ delivered. Figures 1 and 2 display data from one patient enrolled in our trial (ClinicalTrials.gov NCT02871102), which uses a recruitment maneuver and decremental PEEP protocol similar to the ART trial, but with multiple $\mathrm{V}_{\mathrm{T}}$ tested for 2 minutes each at every PEEP level [3]. The curves demonstrate that selection of PEEP by point of maximal compliance or minimal driving pressure varies substantially for a single patient depending on the $\mathrm{V}_{\mathrm{T}}$ with which the best PEEP trial is conducted. This likely occurs due to tidal recruitment.

Our findings suggest one possible mechanism for the ART results. The ART intervention utilized a decremental PEEP trial at a $\mathrm{V}_{\mathrm{T}}$ of $5 \mathrm{ml} / \mathrm{kg}$ predicted body weight (PBW), and then set PEEP at $2 \mathrm{cmH}_{2} \mathrm{O}$ above the PEEP level found to have the maximal $C_{R S}$. If multiple PEEP levels had $C_{R S}$ measures within $1 \mathrm{ml} / \mathrm{cmH}_{2} \mathrm{O}$, the highest PEEP level was chosen. This protocol likely resulted in the use of PEEP levels in the intervention arm associated with a stress index of $>1.05$, suggesting the presence of tidal hyperinflation [4], as demonstrated in our patient by the vertical lines in Fig. 1. Moreover, the curves predict an even greater degree of tidal hyperinflation for patients whose highest PEEP based on maximal compliance was set at one $V_{T}$, but who then received a higher $V_{T}$ for clinical management. Indeed, in the ART trial, intervention patients had a day 1 mean $\mathrm{V}_{\mathrm{T}}$ of $5.6 \mathrm{ml} / \mathrm{kg} \mathrm{PBW}$, implying that many received a $V_{T}$ above that used to select the optimum PEEP.

How to individualize PEEP for patients with ARDS remains a conundrum. Our finding is important because it implies that carefully titrated PEEP may not apply outside of the ventilator parameters with which PEEP was tested and that changes in tidal volume likely influence optimal PEEP.

\footnotetext{
* Correspondence: andrew.c.mckown@vanderbilt.edu 


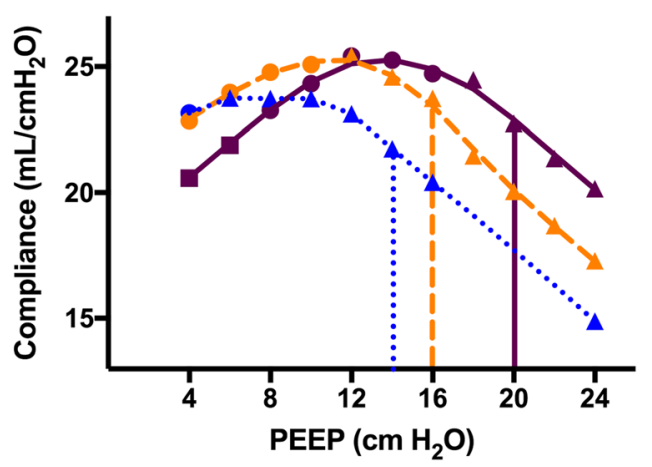

- $4 \mathrm{ml} / \mathrm{kg}$ PBW

$--6 \mathrm{ml} / \mathrm{kg}$ PBW

… $8 \mathrm{ml} / \mathrm{kg}$ PBW

- Stress Index $<0.95$

- $0.95<$ Stress Index $<1.05$

- Stress Index $>1.05$

Fig. 1 Point of maximal compliance depends on tidal volume used in PEEP trial. Plotted markers represent static compliance of respiratory system $\left(C_{R S}\right)$ at each PEEP level during a decremental PEEP trial for a single patient. Marker shapes correspond to measured stress index for the PEEP-tidal volume pairing, and marker color corresponds to tidal volume. Loess curve connects points with identical tidal volumes. Vertical lines indicate the PEEP selected by ART protocol for a given tidal volume. PBW predicted body weight, PEEP positive end-expiratory pressure
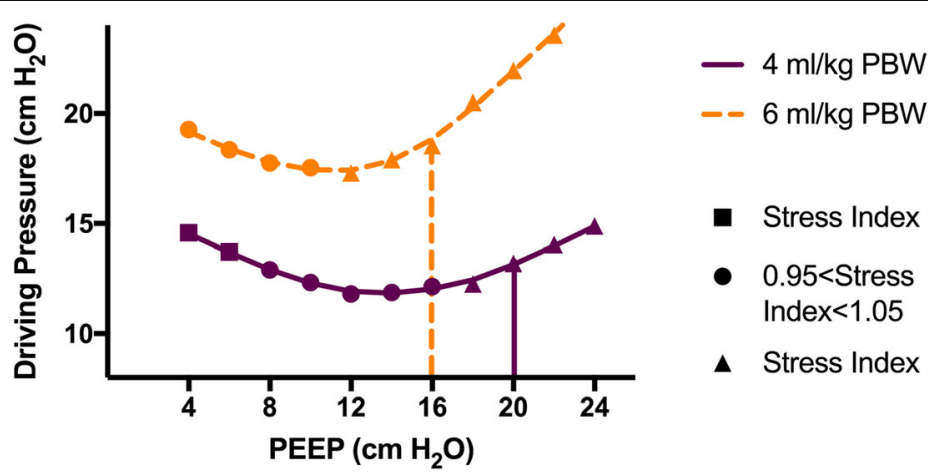

$--6 \mathrm{ml} / \mathrm{kg}$ PBW

- Stress Index $<0.95$

- $0.95<$ Stress Index $<1.05$

- Stress Index $>1.05$

Fig. 2 Point of minimal driving pressure depends on tidal volume used in PEEP trial. Plotted markers are measured driving pressure at each PEEP level for a given tidal volume. Marker shapes coded by stress index, and superimposed loess lines show estimated driving pressure over the range of PEEP by tidal volume. Corresponding vertical lines indicate the PEEP selected by ART protocol for a given tidal volume. PBW predicted body weight, PEEP positive end-expiratory pressure

\section{Funding}

ACM was supported by the Vanderbilt Institute for Clinical and Translational Research (UL1 TR000445 from NCATS/NIH). MWS was supported by a National Heart, Lung, and Blood Institute (NHLBI) K12 award (K12HL133117). ACM and TWR received nonmonetary support from Maquet in the form of access to ServoTracker software and the ventilator Stress Index module for research purposes only. The funding institutions had no role in the conception, design, or conduct of the study; the collection, management, analysis, interpretation, or presentation of the data; or the preparation of the manuscript.

\section{Availability of data and materials}

The datasets generated during the current study are available from the corresponding author on reasonable request.

\section{Authors' contributions}

ACM, MWS, and TWR contributed to the study concept and design. ACM was responsible for acquisition of data. ACM, MWS, and TWR analyzed and interpreted the data. ACM and MWS drafted the manuscript. TWR critically revised the manuscript for important intellectual content. All authors read and approved the final manuscript.
Ethics approval and consent to participate

The study was approved by the Vanderbilt University Institutional Review Board (IRB\# 160770) with informed consent obtained by patient surrogate.

\section{Competing interests}

Maquet was given the opportunity to review the manuscript prior to submission to ensure the absence of Maquet Confidential Information. The authors declare that they have no potential conflicts of interest.

\section{Publisher's Note}

Springer Nature remains neutral with regard to jurisdictional claims in published maps and institutional affiliations.

Received: 11 April 2018 Accepted: 17 April 2018

Published online: 02 May 2018

\section{References}

1. Slutsky AS, Ranieri VM. Ventilator-induced lung injury. N Engl J Med. 2013; 369:2126-36.

2. Sahetya SK, Goligher EC, Brower RG. Fifty years of research in ARDS. Setting positive end-expiratory pressure in the acute respiratory distress syndrome. Am J Respir Crit Care Med. 2017;195:1429-38. 
3. Cavalcanti $A B$, Suzumura ÉA, Laranjeira $L N$, et al; Writing Group for the Alveolar Recruitment for Acute Respiratory Distress Syndrome Trial (ART) Investigators. Effect of lung recruitment and titrated positive end-expiratory pressure (PEEP) vs low PEEP on mortality in patients with acute respiratory distress syndrome: a randomized clinical trial. JAMA 2017;318:1335-1345.

4. Terragni PP, Filippini C, Slutsky AS, Birocco A, Tenaglia T, Grasso S, Stripoli T, Pasero D, Urbino R, Fanelli V, et al. Accuracy of plateau pressure and stress index to identify injurious ventilation in patients with acute respiratory distress syndrome. Anesthesiology. 2013;119:880-9. 